This document is published in:

IEEE Transactions on Systems, Man, and Cybernetics, Part C: Applications and Reviews (2012), 42(4), 561-570.

DOI: 10.1109/TSMCC.2011.2159201

(C) 2012 IEEE. Personal use of this material is permitted. Permission from IEEE must be obtained for all other uses, in any current or future media, including reprinting/ republishing this material for advertising or promotional purposes, creating new collective works, for resale or redistribution to servers or lists, or reuse of any copyrighted component of this work in other works.

This work has been supported by the CAM Project S2009/DPI-1559/ROBOCITY2030 II 


\title{
Personal Autonomy Rehabilitation in Home Environments by a Portable Assistive Robot
}

\author{
Alberto Jardón Huete, Juan G. Victores, Santiago Martínez, Antonio Giménez, and Carlos Balaguer, Member, IEEE \\ A. J. Huete, J. G. Victores, S. Martínez, and C. Balaguer are Robotics Lab Research Group, Department of Systems Engineering and Automation, Universidad \\ Carlos III de Madrid, 28911 Leganes, Spain (e-mail: ajardon@ing.uc3m.es; scasa@ing.uc3m.es; jcgvicto@ing.uc3m.es; balaguer@ing.uc3m.es). \\ A. Giménez is with the Department of Mechanical Engineering, Universidad de Almería, 04120 Almeria, Spain (e-mail: agimfer@ual.es).
}

\begin{abstract}
Increasingly disabled and elderly people with mobility problems want to live autonomously in their home environment. They are motivated to use robotic aids to perform tasks by themselves, avoiding permanent nurse or family assistant supervision. They must find means to rehabilitate their abilities to perform daily life activities (DLAs), such as eating, shaving, or drinking. These means may be provided by robotic aids that incorporate possibilities and methods to accomplish common tasks, aiding the user in recovery of partial or complete autonomy. Results are highly conditioned by the system's usability and potential. The developed portable assistive robot ASIBOT helps users perform most of these tasks in common living environments. Minimum adaptations are needed to provide the robot with mobility throughout the environment. The robot can autonomously climb from one surface to another, fixing itself to the best place to perform each task. When the robot is attached to its wheelchair, it can move along with it as a bundle. This paper presents the work performed with the ASIBOT in the area of rehabilitation robotics. First, a brief description of the ASIBOT system is given. A description of tests that have been performed with the robot and several impaired users is given. Insight into how these experiences have influenced our research efforts, especially, in home environments, is also included. A description of the test bed that has been developed to continue research on performing DLAs by the use of robotic aids, a kitchen environment, is given. Relevant conclusions are also included.
\end{abstract}

Keywords-Climbing robots, clinical trials, homecare, inclusive technologies, portable robots, rehabilitation robotics.

\section{INTRODUCTION}

Most human manipulation tasks require the use of the upper limbs. Because of this, any deficiency in them causes a loss of dexterity and less performance in manipulation. Among disabled people, people with difficulties to use arms and hands are a very representative group. These difficulties affect common DLAs that involve manipulation, such as to move objects, to use tools or utensils, to manipulate small objects, washing, to get dressed, to open and close doors and drawers, to turn switches $\mathrm{ON}$ and $\mathrm{OFF}$, to eat, or to drink.

Technical aids are very useful for psychological rehabilitation and personal motivation, which may be achieved by means of training to perform simple activities with minimal or no external help. Assistive robots have the ability to help people and are capable of providing personalized assistance, individually or in teams [1]. They act as specialized people to help, support, and monitor people with needs, giving them daily independence [2], [3]. During these past 15 years, robotic technology has been evolving to become more flexible and adaptable toward human rehabilitation technique.
Assistive robots are made to help people, to support disabled and elderly people with special needs, inside their own homes and everyday environment.

Traditional rehabilitation robotics technology has been focused on three main development concepts: static systems that operate in structured environments, wheelchair-mounted robotics systems, and mobile manipulator companions capable of following the user for personal and care applications.

The first type of robotic system is very useful for people who need assistance in a reduced part of a living environment and for fixed set of applications, such as eating or drinking. The Handy 1 Robot Arm [4] is an excellent example of a static robot system. It is a low-cost solution for personal care and assistance. Over 400 units have been placed in the market to date. Nevertheless, static robot systems have one major limitation: to change their location can be difficult and may sometimes be near impossible. Using the robot for shaving in a bathroom and eating on another floor could mean having to carry the robot up and down the stairs and fixing and unfixing it manually very frequently. Another inconvenience of static robots is that they intrinsically have limited maneuverability and dexterity due to their static base positioning.

Another type of rehabilitation robots is the wheelchairmounted type. The current market leader of this type of robot is the MANUS system [5]. More than 60 units have been placed to date. The arm is permanently fixed to either the left- or righthand side of the wheelchair. This fixed asymmetry may be of inconvenience for the execution of certain tasks. Additionally, the "bundled" concept implementation may produce mobility problems through doors and stairs. Moreover, the cost of this kind of systems is usually very high.

The third concept suggests a mobile manipulator that follows the user's wheelchair in a structured environment. This concept has disadvantages similar to those of the previous ones. Mobility around a domestic environment is not always ideal due to steps or obstacles. Nevertheless, this concept introduces one new great advantage: The robot has the ability to move around the environment, with independence from the wheelchair or the user. A popular example of this kind of robotic system is the KARES II mobile manipulator [6]. 


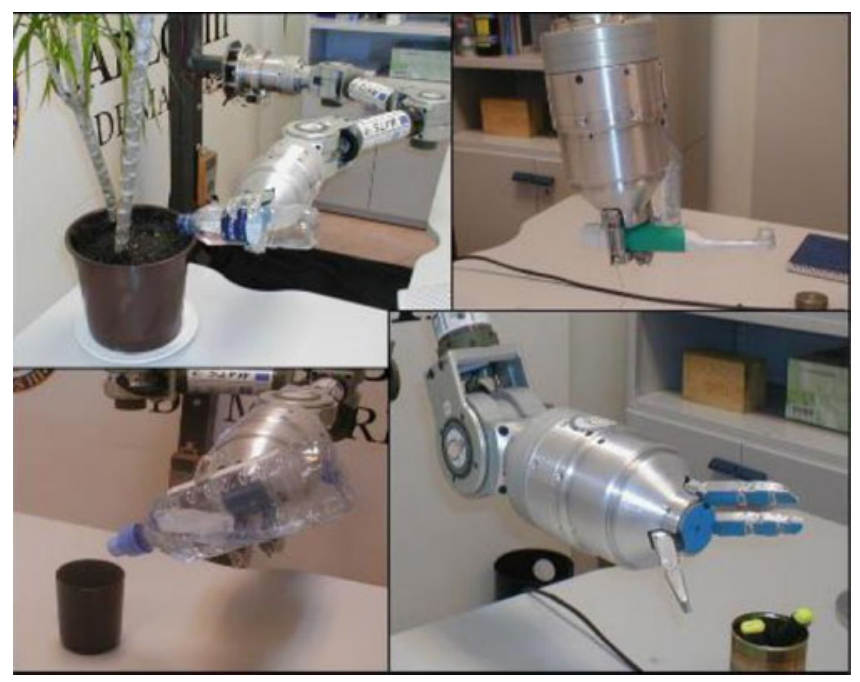

Fig. 1. Assistive robot ASIBOT is portable and has multiple abilities.

The assistive robot ASIBOT (see Fig. 1) has been developed by the Robotics Lab research group at Universidad Carlos III de Madrid (UC3M), Getafe, Spain, and has been endowed with a series of advantageous characteristics, which are unprecedented in other assistive robots. It introduces several useful and unique features.

1) Light-weight symmetrical structure for climbing.

2) Full on-board robot control and communication systems.

3) Unlimited workspace through $24-\mathrm{V}$ climbing connectors.

4) Tool exchange system for grippers, utensils, sponge, etc.

5) Portable and friendly human-machine interface (HMI) adapted to different levels of user capabilities and preferences.

6) Control architecture for integration with environment.

This paper consists of a technical description of the assistive robot ASIBOT and details about experimental results in real domestic environments. Procedures and results of tests in hospital and real domestic environments (a bathroom and a kitchen) will be presented. These trials are not just test simulations or laboratory experiments; they represent a significant advance in assistive robotic application science working with real patients. Tests are focused on to determine the end user acceptability by the use of the robot's unique features and interfaces, therefore differing from clinical trials that are performed with Raptor or MANUS bundled-type systems, which have been evaluated in tests involving groups that are composed of a similar amount of potential end users [7], [8] by different institutions.

\section{Asibot Portable And Personal Robot}

We have managed to introduce climbing and manipulator arm technology into a new robot design with the purpose to assist disabled people. The ASIBOT assistive robot extends human capabilities providing them a way to recover partial autonomy, performing a large variety of domestic operations:

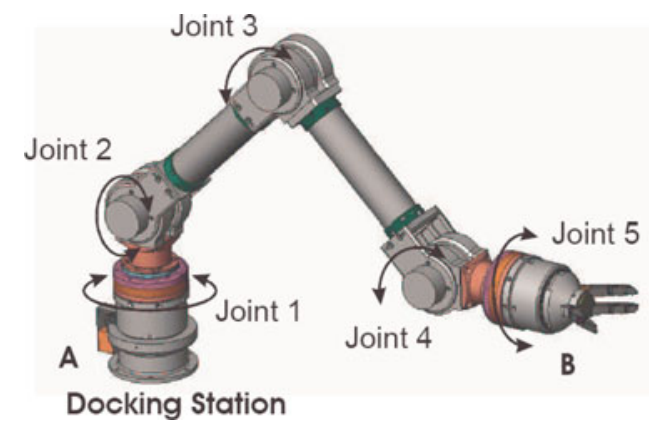

Fig. 2. ASIBOT 3-D representation: DS on one side and gripper on opposite.

housekeeping, self-care, entertainment, etc. It is actually designed to fit into any environment. The robot can move accurately and reliably between rooms and up- or downstairs and can transfer from the wheelchair to floor, ceiling, or wall. This degree of flexibility has significant implications for personal assistance in domestic environments.

\section{A. ASIBOT Portable Design and Climbing Abilities}

ASIBOT is a portable five-degree-of-freedom (5-DOF) manipulator arm (see Fig. 2). Its design is symmetrical and composed of two main parts: the articulated arm structure and the two tips. The articulated body contains two links inside of which all of the electronic equipment and the control unit of the arm are embedded. Each one of the two tips is able to perform two very different functionalities. A tip can connect to a docking station (DS, a climbing connector that provides $24-\mathrm{V}$ power supply) and act as a base for the robot, or be free to perform manipulation tasks. It is important to note that the robot arm's symmetry allows properties, such as the kinematic chain description and, therefore, maneuverability to be (in theory and in practice) independent of which tip is being used as the robot base.

The portability of ASIBOT is achieved due to its light weight. As a $12 \mathrm{~kg}$ manipulator with a $1.3 \mathrm{~m}$ reach, its weight/length ratio is extremely low compared with other manipulator arms, even without considering all of its control systems are on-board. Its payload at tip ranges approximately $2 \mathrm{~kg}$. Communication with the robot is performed wirelessly, through a $802.11 \mathrm{~b}$ secured local area network.

As previously mentioned, the ASIBOT robot can climb from one location to another by attaching its tips to the environment. This moving concept is similar to the CMU's SM2 robot, which uses grippers to attach itself to the space structure [9]. It is also similar to the UC3M ROMA robot that is developed earlier by some of the authors of this paper [10]. ASIBOT's unique feature, however, is its ability to attach itself to the environment (or wheelchair) by the use of especially designed low-cost DSs, which allow it to maintain its manipulation skills. DSs are placed to supply power to the robot allowing it to move and work throughout the entire environment. When a DS is incorporated to a wheelchair, where batteries are available, 24-V power supply may be provided from a direct connection from the batteries to the DS. This concept has been implemented on the wheelchair 


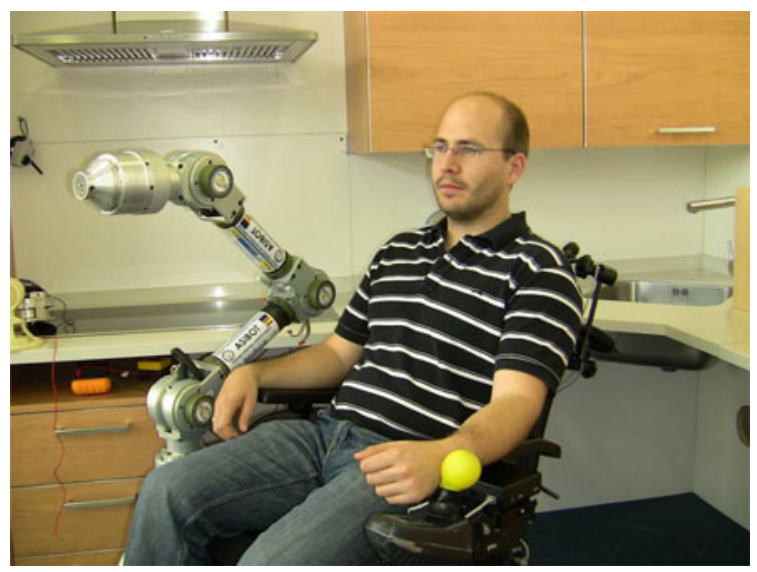

Fig. 3. ASIBOT assistive robot that is fixed on a wheelchair's installed DS.
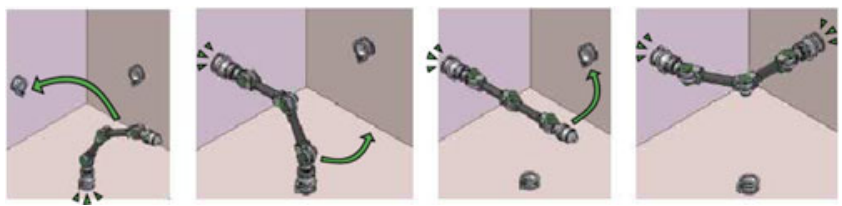

Fig. 4. ASIBOT robot 3-D climbing ability: moving sequence.

that is shown in Fig. 3. This way, even outdoor tasks can be achieved while battery autonomy lasts.

Indoors, a DS may be placed on walls, ceilings, or be furniture mounted. Walls and ceilings are actually recommended places for a DS, since in most cases these spaces are underutilized. This way, the floor remains free, which let wheelchair users to move easily around the environment. Because of the robot's light weight, usually no special modifications are needed to fix DSs on walls or furniture.

As mentioned in Section I, the majority of the mobile assistive robots that have been developed navigate in 2-D indoor environments. They lack DOF and are mounted on the user's wheelchair, like MANUS, or they are fixed to rails as RAID [11], which occupies a lot of floor surface. The ASIBOT robot can virtually increase its DOF by moving in 3-D space, from DS to DS. Fig. 4 shows an ASIBOT climbing sequence, where the robot performs transitions from one surface to another, passing through being attached to three different perpendicular planes [12].

This climbing ability is achieved through the successive fixation and release of tips at DSs. Software permanently assures that at least one of the tips of the robot is docked at a time.

\section{B. ASIBOT End Effectors}

Each tip of the robot is in fact a special male conical connector. DSs, on the other hand, are actually female static conical connectors and are provided with a bayonet locking mechanism (see Fig. 5) that rigidly fixes the robot.

Inside each male connector, a gripper lies that is able to manipulate objects. It is in fact a three-fingered hand with seven phalanges per finger. Fingers are hidden at docking position (the initial state of the sequence that is represented in Fig. 6) and can be released (complete sequence that is represented in the

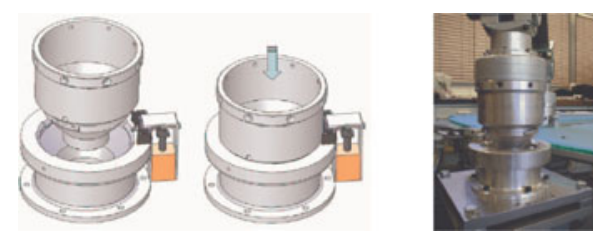

Fig. 5. ASIBOT docking mechanism: tip inserted and locked into a DS.

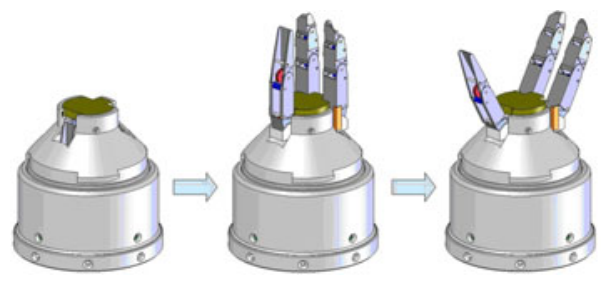

Fig. 6. ASIBOT gripper: sequence from the hidden to the released state.

figure) at will. Tendons drive all of the joints together and allow shape adaptation for grasping [13], [14]. They are activated by a single internal motor that is controlled by its corresponding controller area network (CAN) node. Despite this underactuated and complex design, reduced grasping capabilities are provided, which are capable of grasping many domestic objects within the robot's 2-kg payload limitation.

The end effectors of the ASIBOT are currently under redesign in order to find a more economical and robust solution. Human-like manipulation strategies that are implemented onboard would be very complex to achieve due to mechanical and control issues that are needed for autonomous or partially assisted grasp planning and execution. In addition to common challenges for robotic hand design, an ASIBOT gripper must always have to additionally achieve mechanical fixing in the DS and connect electrical contacts for power supply. This is to comply with critical restrictions that are present in any type of climbing robot: gripping and providing power.

However, robotic restrictions and design issues are not important from the point of view of end users. Practical alternative solutions must be provided. User's demands are related to the usability and the total price of the system. As an alternative, an extensive set of low-cost tools has been designed and manufactured. Rapid prototyping techniques have been used to adapt common household-environment-related tools. These adaptations have been tested by potential end users and are fully compatible with the existing ASIBOT gripper in its hidden position. Fig. 7 shows some of these adapted tools: spoon and cup adaptations for eating and drinking assistance and toothbrush and makeup adaptations for bathroom assistance. All of these adaptations are low cost and functional.

\section{ASIBOT Human-Machine Interface and User Profile}

A fundamental aspect of ASIBOT is its portability. Because of its light weight, it has the capability to be moved by a single person without aid and be carried from one place to another easily. As a climbing robot, all hardware and electronics are on-board to avoid cumbersome "umbilical" wires. One more 


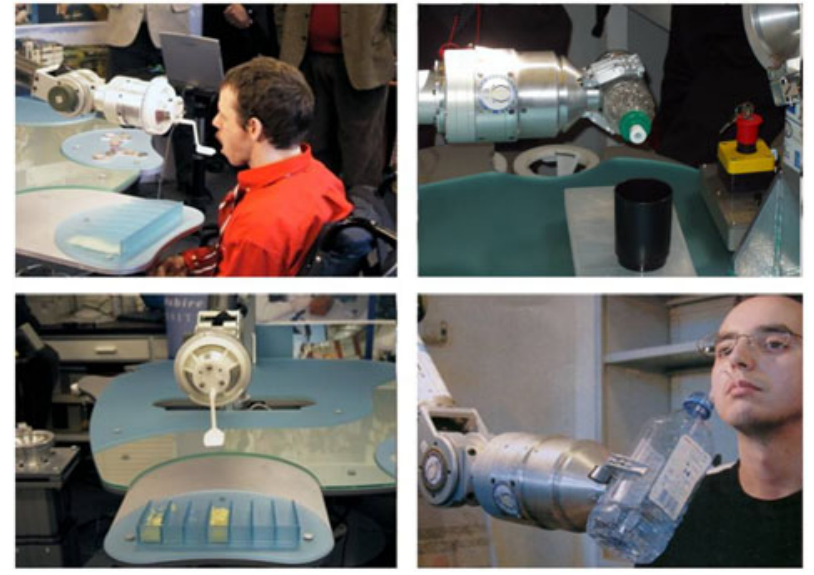

Fig. 7. ASIBOT can use a variety of tools that are adapted to user's needs.

fundamental element exists to promote and maintain portability: the ASIBOT's HMI is also portable.

The control system that is given to the user is based on a multimodal interface that has been developed and compiled to work on a personal digital assistant (PDA) to provide an assisted teleoperation system [15]. The provided multimodal interfaces to control the robot include: tactile screen, using a pointer or a finger, using a scanning system and a button to select options, attaching a joystick, and a voice-recognition system. It is also possible to combine some of these control modes in order to adapt the interface as much as possible to the specific needs of different users. The ASIBOT HMI can be configured to use external joysticks as user-activity transducers (see Fig. 8, all except top right), or to use the wheelchair's driving joystick in order to reduce the number of used control devices.

This user-oriented HMI is designed to control the robot in two different modes: preprogrammed movement mode and usercontrolled robot movement mode. By the usage of the former control mode, only objects (i.e., dishes) that are placed precisely at predefined positions can be manipulated, whereas, in the latter mode, control is completely delegated to the user, and movements in the entire workspace of the robot arm are allowed (control submodes include joint space and Cartesian space). The screenshot of the HMI display unit that is presented in Fig. 8 (top right) corresponds to the preprogrammed movement control mode. The user-controlled robot movement control mode is available through most of the presented multimodal HMI devices. The user-controlled robot movement control mode allows compliance with uncertainty, but task execution becomes tedious for the nonexpert. Execution of preprogrammed tasks is much faster, yet such systems cannot meet with all of the users' requirements. Additionally, the effort required to program tasks has been criticized. A need has been marked for a nontechnically oriented person to be provided with easy tools to perform and program tasks. The conflicting constraints are to maximize flexibility, while minimizing the amount of time it takes to perform a task. Special attention is paid to the variety and diversity of possible users and interaction devices, as overall system performance is HMI dependant, and usability plays a fundamental
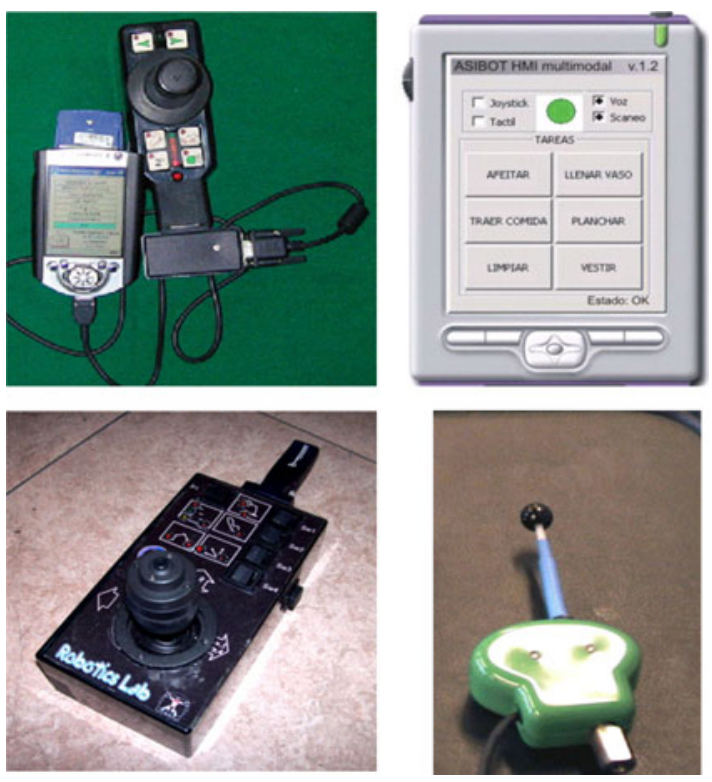

Fig. 8. Different ASIBOT's multimodal HMI devices.

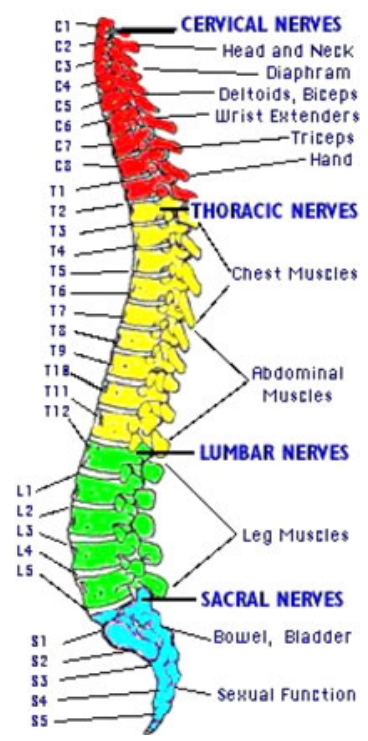

Fig. 9. Location of injury effects is important when predicting which parts of the body might be affected by paralysis and loss of function.

role in the HMI design. Potential users are very limited in the ways in which they can interact with devices.

Cervical (neck) injuries usually result in full or partial tetraplegia (quadraplegia). Depending on the exact location of the injury, a spinal-cord-injured person at cervical level may retain some amount of function (as detailed later), but is otherwise completely paralyzed. Fig. 9 illustrates the specific affected spinal-cord regions. The scale that is referred to in this figure and throughout the rest the paper is the following.

1) C3 vertebrae and above: Typical loss of diaphragm function and require a ventilator to breathe.

2) C4: May retain some use of biceps and shoulders, but weaker. 


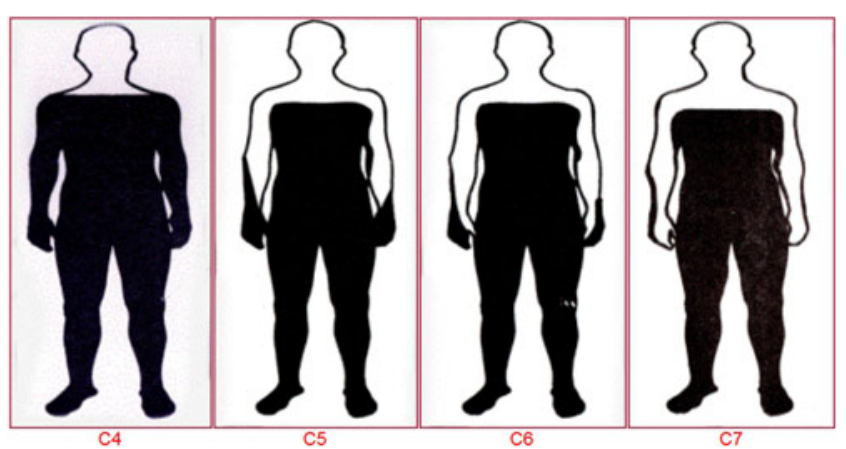

Fig. 10. Lack of sensorimotor functionality is represented in black.

3) C5: May retain the use of shoulders and biceps, but not of wrists or hands.

4) C6: Generally retain some wrist control, but no hand function.

5) C7 and T1: Can usually straighten their arms but still may have dexterity problems with hands and fingers. C7 is generally the level for functional independence, because the user will be able to control a wheelchair.

A person with an incomplete injury retains some sensation or movement below the level of the injury. And, while less than $5 \%$ of people with "complete" spinal-cord injury recover locomotion, over $95 \%$ of people with "incomplete" spinal-cord injury recover some locomotory ability. Fig. 10 shows which parts of the body may be affected by paralysis and loss of function in the case of full spinal-cord injury. An initial study suggested the most suitable potential users that the ASIBOT robot could address, ranging from $\mathrm{C} 2$ to $\mathrm{C} 7$.

On the other hand, interface device specification is variable. Interaction devices address several tradeoffs and complications, making some devices mutually exclusive. A thorough analysis of several HMI techniques can be found in the literature [16]. Nevertheless, Table I shows a list of interface devices versus upper-limb mobility, from different motion-impaired levels and residuals. Each column shows a group of target users, and rows show the usability of several kinds of interface devices. Mobility capacities are ordered from left to right in ascendant order of disability, from those users that are able to move lower limbs, to those with a high degree of motion impairment. The letter in the first column refers to the output format of the device actuated by the user. The nomenclature used in this column is the following.

1) ' $\mathrm{C}$ ' represents a command-type output, which is generated by software running on the PC, PDA, or any mechatronic device that is able to generate high-level protocol commands.

2) 'O' refers to simple devices, such as switches, licorns, or pushbuttons that are physically connected to a control unit (PC, PDA, or similar).

3) 'P' refers to all analog, transducer-based devices, such as joysticks. Devices that are activated by a single hand or foot, chin, back of neck, etc., in which a proportional control requires dexterous control of the related movement, are included in this category.
TABLE I

INTERFACE VERSUS DISABILITY CLASSIFICATION

\begin{tabular}{|l|l|l|l|l|l|}
\hline \multicolumn{1}{|c|}{$\begin{array}{c}\text { Affected } \\
\text { vs. }\end{array}$} & $\begin{array}{l}\text { No leg } \\
\text { mobility }\end{array}$ & $\begin{array}{l}\text { No } \\
\text { upper } \\
\text { nor } \\
\text { lower } \\
\text { limbs }\end{array}$ & $\begin{array}{l}\text { No } \\
\text { head, } \\
\text { neck } \\
\text { nor feet } \\
\text { mobility }\end{array}$ & $\begin{array}{l}\text { Only } \\
\text { vision, } \\
\text { hearing, } \\
\text { and } \\
\text { voice }\end{array}$ & $\begin{array}{l}\text { Only } \\
\text { vision } \\
\text { and } \\
\text { hearing }\end{array}$ \\
\hline $\begin{array}{l}\text { PC, keyboard, } \\
\text { and mouse (C) }\end{array}$ & Yes & No & No & No & No \\
\hline $\begin{array}{l}\text { PDA pointer } \\
\text { (C) }\end{array}$ & Yes & No & No & No & No \\
\hline $\begin{array}{l}\text { PDA + tactile } \\
\text { screen (C) }\end{array}$ & Yes & No & No & No & No \\
\hline $\begin{array}{l}\text { 2D/3D hand } \\
\text { joysticks (P/C) }\end{array}$ & Yes & No & No & No & No \\
\hline $\begin{array}{l}\text { Tactile input/ } \\
\text { haptic output } \\
\text { devices (P/C) }\end{array}$ & Yes & No & No & No & No \\
\hline $\begin{array}{l}\text { Single switch } \\
\text { handled screen } \\
\text { interfaces (O) }\end{array}$ & Yes & Yes & No & No & No \\
\hline $\begin{array}{l}\text { Head, shoulder } \\
\text { or hand gesture } \\
\text { recognition (C) }\end{array}$ & Yes & Yes & Yes & No & No \\
\hline $\begin{array}{l}\text { Head/shoulder } \\
\text { activated } \\
\text { Joysticks (P/C) }\end{array}$ & No & Yes & No & No & No \\
\hline $\begin{array}{l}\text { EMG, Eyes or } \\
\text { gaze tracking } \\
\text { (P/C) }\end{array}$ & Yes & Yes & Yes & Yes & Yes \\
\hline EEG-BCI (C) & Yes & Yes & Yes & Yes & Yes \\
\hline $\begin{array}{l}\text { Facial } \\
\text { command } \\
\text { recognition (C) }\end{array}$ & Yes & Yes & Yes & Yes & Yes \\
\hline
\end{tabular}

However, dependability of a complete system for humanrobot cooperation is dominated by the safety issues. Taking this into account, the simplest solution to cover the users' needs and expectations should be considered the most reliable and useful for the physically impaired. For the sake of user safety, we assume that the user is always in the control loop, at least with the role of supervisor, and can override the current control actions in case he or she is not satisfied with the system behavior.

\section{ASIBOT Safety}

Safety issues are a key factor in rehabilitation robotics. Assistive robots need to operate close and, sometimes, in contact with humans. The ASIBOT safety strategy is based on the following set of factors.

1) Velocity control: The robot velocity is limited on each axis controller and adjusted to achieve a balanced tradeoff between fast transition movements and slow actions when near the user.

2) Safety under power off: The entire system is prepared to be safe during power off, thanks to the motor brakes and the DSs passive design, as no energy is required for a tip to remain attached to a DS.

3) Software safety motion control: Software implements a dead-man-switch (DMS) feature by default, for the sake of the user's safety. Any physical movement of the arm, either in preprogrammed tasks or in direct control, requires the DMS to be kept pressed to be performed. Any time the user relieves pressure from the DMS, the movement 
stops. Other safety measures include security volumes (the zone that is defined around the user, where the robot can never pass, and therefore, undesired collision is avoided), and the robot's base locking system that assures that it is impossible to release one docking mechanism until the next one is safely locked to the DS.

The tradeoff between safety and performance is the key issue in the domain of physical human-robot interaction (pHRI). In the pHRI, accurate positioning is secondary to "natural" soft interaction. Additionally, in assistive devices, time to perform a task is not so critical: slow motions are welcome. As an assistive robot working in cooperation with humans, priority is to assure a totally secure head injury coefficient [17]. As a climbing robot itself, safety must be guaranteed by the consideration that the force of gravity on the robot depends on its position: on the wall, on the ceiling, or on the table. The robot must be safe in every $3-\mathrm{D}$ position in the environment.

Because of the complexity of DLA execution in unstructured and dynamic domestic environments, and the unpredictable or unexpected behavior of a user during task execution, safety must also be granted by means of mechanically safe robots. The limitation in the overall weight and inertia is a common design point for climbing robots. This factor is intrinsic in ASIBOT's design, which provides extra security.

\section{Tests With IMPAiRed USERS AT Hospital NACIONAL DE PARAPLÉJICOS DE TOLEDO}

This part of the paper presents a usability assessment of ASIBOT helping the severely disabled, which is developed as part of the ASIBOT Program at the UC3M in collaboration with the FUHNPAIN, the Foundation for Research and Integration at the National Paraplegic Hospital in Toledo (Hospital Nacional de Parapléjicos de Toledo, HNPT). This is a national reference center that specializes in comprehensive treatment for people with spinal-cord injuries, a physical affliction with several degrees of affection depending on the level and location of the trauma.

Our aim was to gather structured data from the experiment, which reflect opinions about the usage of the robot, focus on the detection of acceptance level, identify prejudices and fears, uncovered needs, and expectations [18], [19]. We also aimed at generation of new ideas from the users' opinions to serve as a base for the improvement of the design of new prototypes.

\section{A. User-Selection Criteria and Pilot Test}

The user-selection criteria was strictly based on rehabilitation doctor expertise. The target population studied was composed of patients who had spinal-cord injuries for at least a year. No cases of patients with extremely acute injuries were considered. Focus was put on users who, once passed an initial phase, had spent regular periods of time in their homes, which gave them a perspective on the main difficulties that they could encounter in their daily lives. Because of their daily experience to face numerous problems of dependence, they were able to evaluate the functionality of technical aids with more objectivity. Only patients that were affected at the cervical level, from neurological levels $\mathrm{C} 4$ to $\mathrm{C} 7$, were chosen, as the resulting limitations affect their upper extremities, yet do not eliminate the possibility of the usage of the different interfaces for the proposed tests. Additional exclusion criteria were as follows: epilepsy, mental retardation, uncorrected visual deficiency, or psychiatric problems. The final group that was selected to perform the tests was composed of the five hospital patients, who fulfilled the described criteria. Regarding the number of users for a proper usability assessment, Virzi [20] and, more recently, Lewis and Turner [21], [22] have published influential articles on the topic of the sample size in usability testing. According to these authors, 5 is a proper number for usability testing.

The experiment procedure included a pilot test prior to the tests in real settings in order to assess different modes of robotuser interaction. These were implemented through a GUI on a PDA, where six different options were given, in the form of large visual-tactile buttons, each to command a different potential robot task to be performed: shave, fill a cup, feed, iron, clean, dress, etc. The following modes of interaction were implemented (sorted according to the degree of mobility that is required for their use, from most to least).

1) Tactile: task selection via the user's touch or a pencil, double touch to validate.

2) Joystick: movement through joystick for task selection, pressing a button for validation.

3) Voice recognition: tactile or joystick options for task selection, validation by voice recognition.

4) Turning on sequence: the selected task changes automatically every certain time, validation by pressing a button.

These mechanisms were selected as potential interfaces with the robotic system, given they are the most commonly accepted among the user-interface community [23], [24]. The users were, then, asked to give their opinion on each interface, focusing on easiness of use, practicality, and how appropriate they found each interface taking into account their own individual capabilities.

\section{B. Scenario and Task Selection for Tests}

At this stage, the users were queried on which activities they found most unpleasant and would like to be able to perform without depending on another person, regardless of whether the robot could do them or not. Getting dressed and personal hygiene were the most commonly mentioned tasks. Additional mentioned tasks included cleaning the house, cooking, making or unmaking the bed, folding sheets, dressing, and tasks that require additional accuracy, such as shaving, cutting nails, combing hair, picking up glasses, opening windows, and opening doors. The users were, then, asked to set their order of priority on four settings that we proposed. These settings were based on the tasks that they had proposed and basic feasibility factors. The results, from highest priority to lowest, were the following.

1) Personal hygiene: washing one's face and hands, brushing teeth, combing hair, shaving, applying makeup, etc.

2) Lying in bed: bringing small objects near, etc.

3) On the wheelchair: eating, drinking, bringing small objects near, etc. 


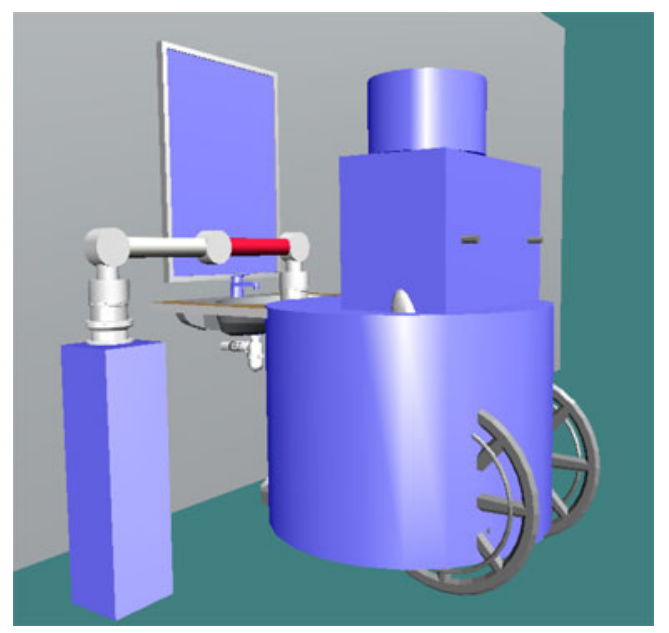

Fig. 11. Bathroom safety-area representation on the 3-D VRML model.

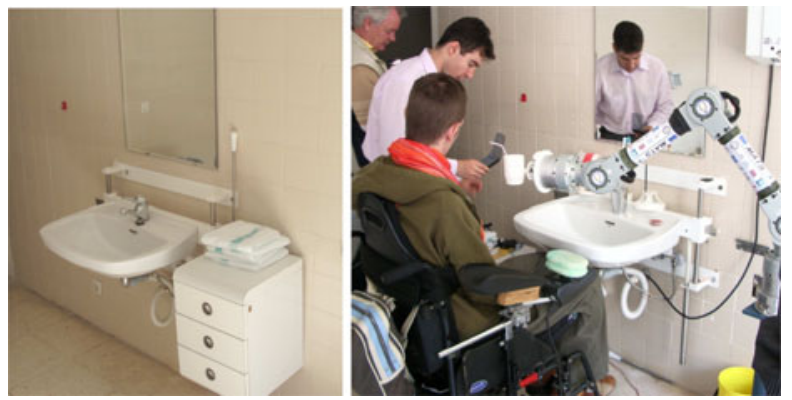

Fig. 12. Bathroom at HNPT's pilot home, before and after adaptations.

4) In the kitchen: opening cupboards, moving utensils, etc.

Given the fact that personal hygiene was the preferred setting for the robot in terms of this priority rating, the selected environment for the tests was a bathroom scenario. Five tasks were selected from the users' proposals, taking into account feasibility, time consumption, and easiness to setup criteria: 1) drinking, 2) brushing one's teeth, 3) putting makeup on or drying one's face, 4) washing one's face, and 5) picking objects up.

\section{Test Setup and Necessary Adaptations}

The adaptations for the selected activity tests at the bathroom scenario were minimal. As a first step, a simulated version of the environment was developed by the use of the MATLAB VRML toolbox. By the usage of this simulated environment, the optimum number of DSs and their location and orientation were determined. Only two DSs were determined to be needed. Additionally, safety areas were set (as shown in Fig. 11), to be used to avoid undesired robot-user and/or robot-environment collision.

The main physical task was the mechanical installation of the DS needed to perform the desired tasks. The two DSs were fixed by the use of a steel structure, locating one at each side of the sink as shown in Fig. 12.

Even though each robot tip has a gripper with three fingers, the shape, size, and texture of an object can make its manipulation very difficult. This is, especially, notable in household environments, such as a bathroom, where common objects, such as soap, are extremely difficult to work with. In order to solve this issue, the rapid-prototyping-adapted tool mechanism was used. Several new tools were developed for the robot, which are meant to be attached by the same bayonet system that is used in the docking process. The following set of adapted tools was developed: an electric shaver, an electric toothbrush, a makeup brush, a cup, a sponge, and a bottle. Fig. 13 depicts some of these adapted ASIBOT tools.

The position over the shelf was selected for tool exchanging, and an automatic soap dispenser for the cleaning task with the sponge tool was installed. Another modification was the substitution of the classic faucet by an automated infrared one. This is an example of a universally accessible product. The robot activates the faucet by approaching with the toothbrush, the same way a user with enough mobility would.

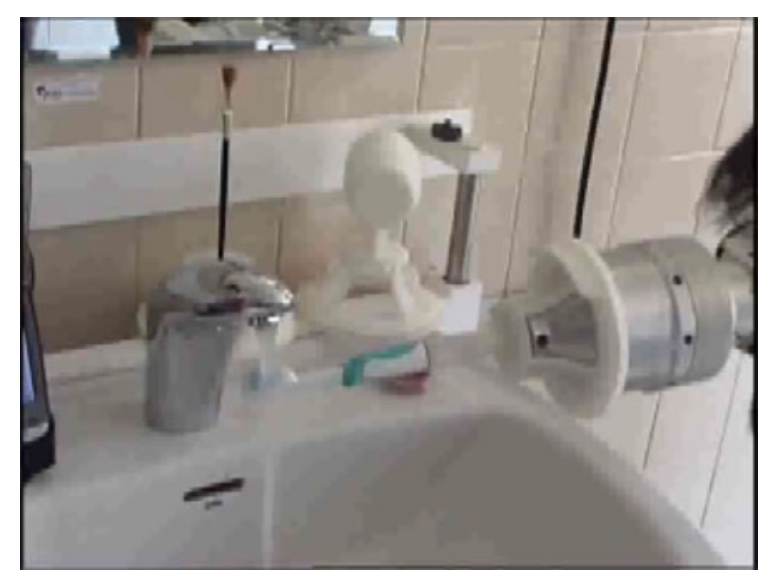

Fig. 13. Toothbrush toolholder in use and cup and makeup brush in background.

\section{Bathroom Scenario Performance Test and Results}

The robot's features and HMI capacity were evaluated individually by each user, who expressed their opinions via a range of satisfaction scores, from -2 to +2 .

Table II summarizes the user's questionnaire and results on specific tasks. It can be seen that simple tasks, such as to give the user something to drink, achieve relatively high rankings. Table III summarizes the user's questionnaire and results on the robot itself. The top ratings refer to the aspects of ease of handling, how quickly it worked, and its multiuse functionality.

The users were, finally, asked what changes they suggest to make the robot more useful. The most common suggestions were a smaller size, greater ease in robot mobility, and complete recognition of the user's natural speech. Moreover, the overall impression was that users significantly appreciated the chance to perform DLAs by themselves using the ASIBOT assistive robot as an aid: partially recovering personal autonomy and gaining self-esteem. 
TABLE II

USERS' QUESTIONNAIRE RESUlTs (MEAN/MODE)

\begin{tabular}{|l|l|l|l|l|l|}
\hline $\begin{array}{l}\text { Tasks (according } \\
\text { to section III-B) }\end{array}$ & (a) & (b) & (c) & (d) & (e) \\
\hline $\begin{array}{l}\text { Does the robot } \\
\text { help you? }\end{array}$ & $0,84 / 2$ & $0,8 / 1$ & $0,8 / 1$ & $1,33 / 2$ & $1,2 / 2$ \\
\hline $\begin{array}{l}\text { Is it useful and } \\
\text { easy to use? }\end{array}$ & $1,2 / 2$ & $0,6 / 1$ & $0,7 / 1$ & $0,66 / 2$ & $0,84 / 1$ \\
\hline $\begin{array}{l}\text { Do you need an- } \\
\text { other aide using } \\
\text { the robot? }\end{array}$ & $0,8 / 1$ & $0,33 / 1$ & $0 /-1$ & $0,14 / 1$ & $1,2 / 1$ \\
\hline $\begin{array}{l}\text { Is the robot able to } \\
\text { increase your au- } \\
\text { tonomy? }\end{array}$ & $1,4 / 1$ & $0,5 / 0$ & $0,2 /-1$ & $0,66 / 2$ & $0,8 / 1$ \\
\hline $\begin{array}{l}\text { Does its use imply } \\
\text { physical or mental } \\
\text { effort? }\end{array}$ & $1,4 / 2$ & $0,66 / 1$ & $0,33 / 1$ & $0,33 / 1$ & $0,5 / 1$ \\
\hline $\begin{array}{l}\text { Does the robot } \\
\text { perform desired } \\
\text { tasks successfully? }\end{array}$ & $1,8 / 2$ & $0,16 / 0$ & $1,66 / 1$ & $1,3 / 1$ & $0,4 / 1$ \\
\hline $\begin{array}{l}\text { Do you feel moti- } \\
\text { vated to use it for } \\
\text { this activity? }\end{array}$ & $1,2 / 1$ & $0,8 / 0$ & $0,7 / 1$ & $0,6 / 1$ & $0,6 / 0$ \\
\hline $\begin{array}{l}\text { Overall impression } \\
\text { Don }\end{array}$ & 2 & 1 & 1 & 1 & 1 \\
\hline
\end{tabular}

TABLE III

USERS' OVERALL VALUATION

\begin{tabular}{|l|l|}
\hline TOPICS & Mean / mode \\
\hline Aesthetic & $0,65 / 2$ \\
\hline Size, Range & $-0.25 / 0$ \\
\hline Easy to use & $0,83 / 1$ \\
\hline Operation Speed & $1,54 / 2$ \\
\hline Safety & $0,82 / 1$ \\
\hline Robustness & $1,85 / 2$ \\
\hline Easy setup & $1,20 / 1$ \\
\hline Overall impression & $1 / 1$ \\
\hline
\end{tabular}

\section{ASIBOT KITCHEN ENVIRONMENT TEST BED}

After having performed the hospital tests, a test bed to continue research on performing DLAs in household environments was developed. This test bed is a kitchen scenario that contains real household appliances, in addition to features that faithfully represent a real home environment that is prepared for impaired users (counter height prepared for use of a wheelchair, automated shelves, presence sensors, etc.). It is essentially a complete living space that is ready to be used by impaired and nonimpaired users. The kitchen, additionally, is portable, as it is made out of demountable assembled modules. It is currently located at UC3M's Technological Centre, more specifically, in the Assistive Robot Laboratory. The kitchen's free surface ranges approximately $19 \mathrm{~m}^{2}$.

The fully furnished kitchen is now provided with fixed and rail-mounted DSs to support ASIBOT's fixation and energy

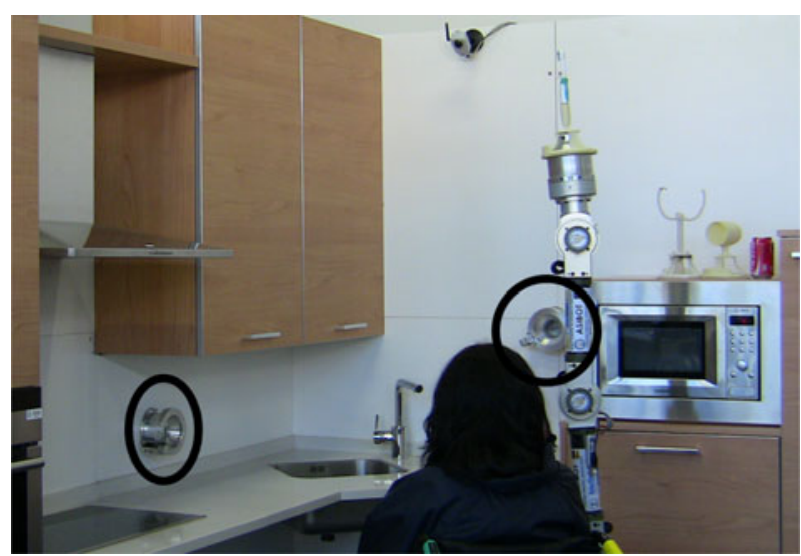

Fig. 14. ASIBOT kitchen environment test bed.

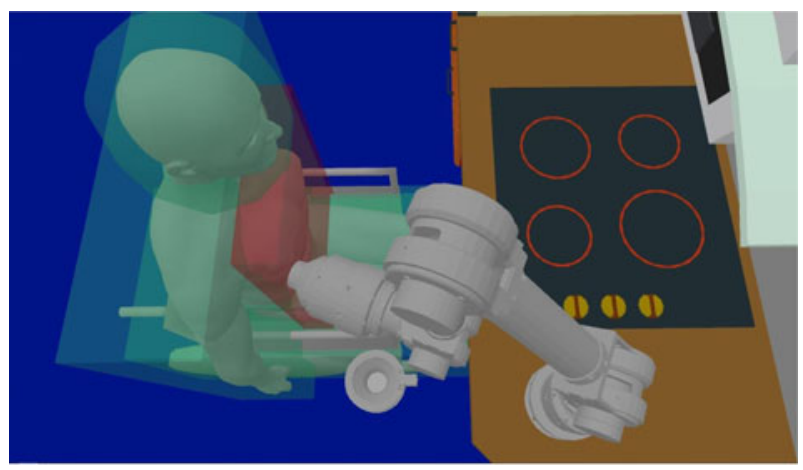

Fig. 15. Scene of collision with user-safety volume that is computed during simulation.

requirements. The number of DSs was minimized while still optimizing the robot reach volume. Two of the total of 6 installed DSs are shown in Fig. 14 (both large circles). The physical installation of DSs among the kitchen has not reduced the kitchen's functionalities.

\section{A. ASIBOT Kitchen Test-Bed Simulation and Integration}

The authors have recently developed a virtual ASIBOT and kitchen test-bed simulation environment. It is based on the Open Robotics Automation Virtual Environment (OpenRAVE) [25] core libraries. The underlying idea is to provide a low-cost virtual environment to allow performing tests with time savings and effectiveness.

It is currently used for research in planning and learning and, in addition, serves as a practical platform for

1) robot online and offline task programming (correction of errors, safety issue testing as provided by virtual safety volumes shown in Fig. 15);

2) robot prototype and tool adaptation design (physical dimensions, weight, manipulability);

3) DS optimizer to determine number, location (on furniture, over the cooking top, ceiling hanged, etc.), and type (fixed, rail mounted, wheelchair mounted, etc.) of DSs;

4) demonstrator for potential users and caregivers. 


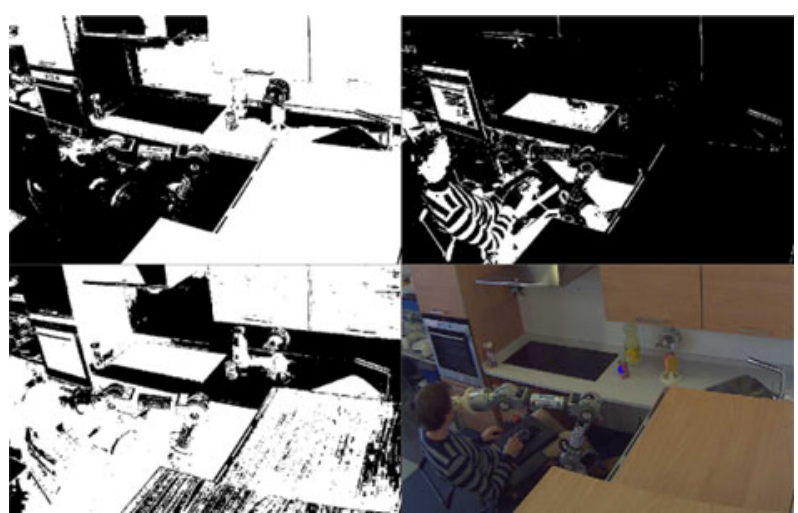

Fig. 16. Color segmentation and object centroid on kitchen IP camera image.

The simulated kitchen environment, HMI devices, and the real robotic system have been integrated into a common software architecture yet another robot platform (YARP) [26]. YARP is used because of its light weight, multiplatform support, simple application programming interface (API), and bindings for many programming languages [27]. Another benefit is that connections are made seamlessly: commands can be sent to the robot, the simulation, or to both (simultaneously) due to the common interface and publisher/subscriber paradigm implementation.

\section{B. Assistive-Living Devices and Sensors}

Standard domotic devices that are connected by an European installation bus (EIB) bus have already been introduced to explore cooperation between assistive devices. They are accessible through a software gateway that communicates with the robot network. Additionally, embedded devices are being adapted to interface kitchen electrical appliances to the common services architecture. Four IP-server surveillance cameras have been set in strategic locations to send raw image data to be processed by the room controller. These devices are linked by exchange services and keep tabs on each other and the user. Photogrammetric algorithms are being applied to discover absolute Cartesian coordinates of objects of interest for the user. Images received from the environmental cameras are processed by color segmentation, and centroids are calculated through standard machine vision algorithms. Full algorithms have recently been published by some authors of this paper [28]. Fig. 16 shows an example of the results of these processes. The absolute Cartesian coordinates of localized objects are transformed into coordinates that are useful to the robot.

\section{CONCLUSION}

Until now, two adapted environments that are specially designed to support motion impaired have been adapted. These scenarios, i.e., a bathroom and a kitchen, have been adapted with minimal modifications: strategically localized DS, and optional toolholders, IP environment cameras, and extra sensors. The first environment to be adapted was the bathroom of the DLA Occupational Therapy apartment located at the Hospital
Nacional de Parapléjicos de Toledo installations. The second one, i.e., a kitchen environment test bed, has been assembled at one of Robotics Lab's laboratories. This place will integrate and establish a common framework for several research groups and University related enterprises that are interested in technology transfer. The future work scenario would coordinate the efforts of many researchers and stakeholders, which are organized in user-targeted multidisciplinary teams, to design, develop, and evaluate technical aids and system. The common objective is to preserve and increase the personal autonomy of its users.

ASIBOT's ability to move around the house between fixed or mobile stations has been deeply tested, and clinical trial has discovered its usefulness to assist motion impaired to perform a wide variety of tasks by themselves: eating, cleaning, washing, handling, etc. However, previous-programmed-based behavior is not enough to meet the user's expectations, and deep usability improvement areas have been detected. The next version of the ASIBOT robotic system aims to be a safe and reliable domestic robot assistant with its mechatronic design, force-torque sensing, cameras at tips, and integrated control along the entire robot structure. Our target is to develop and test a new light-weight domestic climbing robot that is specifically designed and programmed for HRI in domestic environments, "dependability proven" and ready to be used by anyone, in a customizable personal way. Extensive experimental and clinical trials and direct user implications on design stages will be continued to find definitive, widely accepted solutions.

\section{ACKNOWLEDGMENT}

The authors would like to thank the Robotics Lab team, R. Correal, R. Palencia, V. Placer, and many others, for their help to develop the ASIBOT trials. The authors would also like to acknowledge the work of the other partners involved in the projects. 


\section{REFERENCES}

[1] M. Mataric, J. Eriksson, D. J. Feil-Seifer, and C. J. Winstein, “Socially assistive robotics for post-stroke rehabilitation,” J. Neuroeng. Rehabil., vol. 4,no. 1, p. $5,2007$.

[2] A. Jardón, “Assistive robot design methodology: Application to portable robot ASIBOT,” Ph.D. dissertation, Dept. Syst. Eng. Autom., Univ. Carlos III Madrid, Spain, 2006.

[3] A. Jardón, A. Giménez, R. Correal, R. Cabas, S. Martínez, and C. Balaguer, "A portable light-weight climbing robot for personal assistance applications," Ind. Robot., Int. J., vol. 33, no. 4, pp. 303-307, 2006.

[4] M. Topping, "Early experiences encountered in the placement of 60 handy 1 robotic aids to eating," in Proc. IEEE Conf. Cybern., Le Touguet, France, 1993, pp. 543-546.

[5] Exact Dynamics. (Mar. 23, 2011.). [Online]. Available: http://www.exactdynamics.nl/.

[6] Z. Bien, M. Chung, P. Chang, D. Kwon, D. Kim, J. Han, J. Kim, D. Kim, H. Park, S. Kang, K. Lee, and S. Lim. “Integration of a rehabilitation robotic system (KARES II) with human-friendly man-machine interaction units,” in Autonomous Robots, vol. 16. Norwell, MA: Kluwer, 2004, pp. 165-191.

[7] E. Chaves, A. Koontz, S. Garber, R. A. Cooper, and A. L. Williams, "Clinical evaluation of a wheelchair mounted robotic arm," RESNA Presentation given by E. Chaves, 2003.

[8] H. Tijsma, F. Liefhebber, and J. Herder, “Evaluation of new user interface features for the manus robot ARM,” in Proc. IEEE Int. Conf. Rehabil. Robot., 2005, pp. 258-263.

[9] M. Nechyba and Y. Xu, "SM2 for new space station structure: Autonomous locomotion and teleoperation control," in Proc. IEEE Int. Conf. Robot. Autom., San Diego, CA, May 1994, pp. 1765-1770.

[10] C. Balaguer, A. Giménez, and M. Abderrahim, “A climbing autonomous robot for inspection applications in 3-D complex environment,” Robotica, vol. 18, no. 3, pp. 287-297, Sep. 2000 .

[11] J. L. Dallaway and R. D. Jackson, “The RAID workstation for office environments,” in Proc. Rehabil. Eng. Assistive Technol. Soc. North America Annu. Conf., 1993, pp. 504-506.

[12] C. Balaguer, A. Giménez, and A. Jardón, “Climbing robots' mobility for inspection and maintenance of 3D complex environments,” Auton. Robots, vol. 18 , no. 3, pp. $157-169,2005$.

[13] R. Cabas and C. Balaguer, "Design and development of a light weight embodied robotic hand activated with only one actuator," in Proc. IEEE/RSJ Int. Conf. Intell. Robots Syst., Edmonton, Canada, Aug. 2005, pp. 2369-2374.

[14] R. Cabas, L. M. Cabas, A. Jardón, and C. Balaguer, “Optimized design of the underactuated robotic hand,” presented at the Int. Conf. Robot. Autom.,Orlando, FL, May 2006.

[15] C. Balaguer, A. Giménez, A. Jardón, R. Correal, and S. Mart'1nez, “Advances in telerobotics,” in Proprio Teleoperation of a Robotic System for Disabled Persons' Assistance in Domestic Environments, vol. 31. New York: Springer, 2007, pp. 389-402, ISBN: 978-3-540-71363.

[16] M. Busnel, R. Cammoun, F. Coulon-Lauture, J. M. D'etrich, G. Le Claire, and B. Lesigne, "The robotized workstation "MASTER" for users with tetraplegia: Description and evaluation,” J. Rehabil. Res. Develop.,vol. 36, no. 3, pp. 217-229, 1999.

[17] A. Bicchi and G. Tonietti, "Fast and "soft-arm” tactics [robot arm design],” IEEE Robot. Autom. Mag., vol. 11, no. 2, pp. 22-33, Jun. 2004.

[18] L. Demers and B. Ska, "Item analysis of the quebec user evaluation of satisfaction with assistive technology (QUEST)," Assist. Technol., vol. 12, pp. 1296,2000 .

[19] L. Demers et al., "An international content validation of the Quebec user evaluation of satisfaction with assistive technology (QUEST)," Occupat. Therapy Int., vol. 6, no. 3, pp. 159-175, 1999.

[20] R. A. Virzi, "Refining the test phase of usability evaluation: How many subjects is enough?," Human Factors, vol. 34, pp. 457-468, 1992.

[21] J. R. Lewis, "Evaluation of procedures for adjusting problem-discovery rates estimated from small samples," Int. J. Human-Comput. Interact., vol. 13, pp. 445-479, 2001

[22] C. W. Turner, J. R. Lewis, J. Nielsen, “Determining usability test sample size,” International Encyclopedia Ergonomics Human Factors, 2nd ed, vol. 3, pp. 3084-3088, 2006.

[23] H. Eftring, "Robot control methods and results from user trials on the RAID workstation," in Proc. Int. Conf. Rehabil. Robot., 1994 , pp. 97-101.

[24] Z. Han, H. Jiang, P. Scucces, S. Robidoux, and Y. Sun, "PowerScan: A single-switch environmental control system for persons with disabilities," in Proc. 8-9th IEEE Bioeng. Conf., 2000, pp., 171-172.

[25] R. Diankov. (2010, Aug.) Automated Construction of Robotic Manipulation Programs. Carnegie Mellon University, Robotics Institute. [Online]. Available: ttp://openrave.programmingvision.com.

[26] P. Fitzpatrick, G. Metta, and L. Natale, “Towards long-lived robot genes,” Robot. Auton. Syst., vol. 56, no. 1, pp. 29-45, 2008.

[27] J. G. Victores, "Software engineering techniques applied to assistive robotics: Guidelines \& tools," Master thesis, Dept. Syst. Eng. Autom., Univ.Carlos II of Madrid, Spain, Oct. 2010

[28] J. G. Victores, A. Jardón, M. F. Stoelen, S. Martínez, and C. Balaguer, “ASIBOT assistive robot with vision in a domestic environment,” pre-sented at the Robocity2030 7th Workshop, Móstoles, Universidad Rey Juan Carlos, Spain, Oct. 2010, pp. 61-74, Visión en Robótica, ISBN: 84-693-6777-3. 